\title{
LES TMS ET LE MAINTIEN EN EMPLOI DES SALARIÉS DE 50 ANS ET PLUS : UN DÉFI POUR LA SANTÉ AU TRAVAIL ET LA SANTÉ PUBLIQUE
}

Elise Chiron,Yves Roquelaure1,Catherine Ha,Annie Touranchet,Anne Chotard,Patrick Bidron4,François Leroux4,Annick Mazoyer4,Marcel Goldberg,Ellen Imbernon2, Béatrice Ledenvic

\section{S.F.S.P. | « Santé Publique »}

2008/hs Vol. 20 | pages 19 à 28

ISSN 0995-3914

\section{Article disponible en ligne à l'adresse :}

http://www.cairn.info/revue-sante-publique-2008-hs-page-19.htm

\section{Pour citer cet article :}

Elise Chiron et al., «Les TMS et le maintien en emploi des salariés de 50 ans et plus : un défi pour la santé au travail et la santé publique », Santé Publique 2008/hs (Vol. 20), p. 19-28.

Distribution électronique Cairn.info pour S.F.S.P..

(C) S.F.S.P.. Tous droits réservés pour tous pays.

La reproduction ou représentation de cet article, notamment par photocopie, n'est autorisée que dans les limites des conditions générales d'utilisation du site ou, le cas échéant, des conditions générales de la licence souscrite par votre établissement. Toute autre reproduction ou représentation, en tout ou partie, sous quelque forme et de quelque manière que ce soit, est interdite sauf accord préalable et écrit de l'éditeur, en dehors des cas prévus par la législation en vigueur en France. Il est précisé que son stockage dans une base de données est également interdit. 


\title{
Les TMS et le maintien en emploi des salariés de 50 ans et plus : un défi pour la santé au travail et la santé publique
}

MSDs and job security of employees aged 50 years and over:
a challenge for occupational health and public health

\author{
Elise Chiron (1), Yves Roquelaure (1), Catherine Ha (2), Annie Touranchet (3), \\ Anne Chotard (4), Patrick Bidron (4), Béatrice Ledenvic (4), François Leroux (4), \\ Annick Mazoyer ${ }^{(4)}$, Marcel Goldberg ${ }^{(5)}$, Ellen Imbernon (2) \\ et 78 médecins du travail de la région des Pays de la Loire
}

Résumé : Les troubles musculosquelettiques des membres (TMS) sont la première cause de maladie professionnelle en France comme en Europe. Le réseau pilote de surveillance épidémiologique des TMS mis en place par l'Institut de Veille Sanitaire en 2002 dans la région des Pays de la Loire apporte des renseignements inédits sur la prévalence des TMS et de leurs facteurs de risque dans la population salariée, notamment vieillissante. Entre 2002 et 2004, un réseau sentinelle de 83 médecins du travail volontaires a permis d'inclure par tirage au sort 3710 salariés âgés de 20 à 59 ans représentatifs des salariés de la région. Les six principaux TMS des membres supérieurs sont diagnostiqués cliniquement selon un protocole rigoureux. L'évaluation de l'exposition professionnelle s'effectue à l'aide d'un auto-questionnaire portant sur les facteurs biomécaniques et psychosociaux de risque de TMS. Au moins un des six principaux TMS a été diagnostiqué au cours de l'examen clinique chez $15 \%$ des femmes et $11 \%$ des hommes. Parmi les salariés âgés de 50 à 59 ans, près de $19 \%$ des hommes et $27 \%$ des femmes souffrent d'au moins un des six TMS. Les catégories ouvrières sont les plus touchées, quels que soient l'âge et le sexe, devant les employés. L'exposition professionnelle aux facteurs de risque de TMS reste élevée chez les 50-59 ans, quel que soit le sexe : $77 \%$ des ouvriers et $72 \%$ des ouvrières restent exposés à au moins deux des facteurs de risque de TMS. Les implications pour la prévention des TMS et le maintien au travail des salariés sont discutées. La forte prévalence des TMS parmi les 50-59 ans conjuguée à des contraintes biomécaniques et psychosociales importantes souligne la nécessité d'une approche globale de la prévention des TMS dans les entreprises pour réduire l'exposition au risque et assurer le maintien et/ou le retour au travail des salariés souffrant de TMS.

Mots-clés : Troubles musculosquelettiques des membres supérieurs - travailleurs vieillissants politique de santé au travail.

(1) Laboratoire d'Ergonomie et d'Épidémiologie en Santé au Travail, Unité associée InVS, Médecine E - CHU, 4 rue Larrey, 49933 Angers Cedex.

(2) Département Santé Travail, InVS, Saint-Maurice.

(3) DRTEFP Pays de la Loire, Nantes.

(4) Services de santé au travail d’Angers, Le Mans, Laval, Nantes et La Roche-sur-Yon.

(5) Inserm U687-IFR 69, Villejuif. 
Summary: Work-related musculoskeletal disorders of the upper limbs (MSDs) are the first cause of occupational illness in France, as in Europe. A pilot network for epidemiological surveillance system of MSDs has been implemented in France's Loire Valley region to assess the prevalence of MSDs and their risk factors in the working population, namely the ageing workforce. The surveillance was based on a network of occupational physicians and used the recommendations of a group of European experts ('Saltsa Criteria document' consensus). Between 2002 and 2004, 3,710 men and women (aged 20-59) representative of the regional workforce were randomly selected. Exposure to risk factors of MSDs was evaluated by the use of a self-administered questionnaire which aimed to assess bio-mechanical and psychosocial risk factors. With at least one of the six primary MSDs of the upper limbs having been clinically diagnosed using a rigorous protocol, the prevalence of clinically-diagnosed MSDs was high: about $11 \%$ of male and $15 \%$ of female workers suffered from at least one of the MSDs studied. After age fifty, about $19 \%$ of male and $27 \%$ of female workers suffered from at least one MSD and $4 \%$ of male and $6 \%$ of female workers from at least two disorders. Blue-collar workers were the most affected, regardless of age or gender, followed by office employees. Exposure to MSD risk factors of remained high after age fifty: $77 \%$ of male and $72 \%$ of female blue-collar workers were exposed to at least two MSD risk factors. The implications for the prevention of MSDs and job security of workers are discussed. The high prevalence of specific MSDs of the upper limb combined with a high exposure to risk factors of MSDs in ageing workers emphasizes the need to implement comprehensive prevention programs in most sectors to reduce the exposure to risk, the prevalence of MSDs and ensure the capacity to keep employees suffering from MSDs working, or at least to quickly return to work after recovery.

Keywords: Upper limb musculoskeletal disorders - epidemiological surveillance - ageing workforce - occupational health policy. 


\section{Introduction}

La dénomination "troubles musculosquelettiques" (TMS) des membres regroupe un ensemble d'affections douloureuses touchant les tissus mous péri articulaires (muscles, tendons, nerfs, etc.) secondaires à l'hyper sollicitation des membres supérieurs et/ou inférieurs d'origine professionnelle $[6,11,14]$. Les affections les plus fréquentes sont, d'une part des maladies bien codifiées comme le syndrome du canal carpien (SCC) ou compression du nerf médian au poignet, l'épicondylite à la face latérale du coude et les tendinopathies de la coiffe des rotateurs (TCR) de l'épaule et, d'autre part, des syndromes douloureux non spécifiques.

Les TMS constituent l'une des questions les plus préoccupantes en santé au travail et santé publique $[1,6,10]$ : ils représentent un coût humain et socioprofessionnel considérable en termes de douleurs et de gênes dans le travail et de la vie quotidienne, de séquelles fonctionnelles parfois irréversibles, de réduction d'aptitude au travail et de risque de rupture de carrière professionnelle. Ils sont la première cause de maladies professionnelles (MPI), avec plus de 23000 cas indemnisées en France en 2003 dont plus de 9000 avec séquelles [3].

Le coût global des TMS pour la société est considérable et avoisinerait, selon l'Agence européenne pour la santé et la sécurité au travail, 1,5 \% du PIB européen [1,6]. Il s'agit d'un des enjeux majeurs de la santé au travail dans les dix années à venir, en raison de leur probable augmentation liée à l'intensification des conditions de travail et du vieillissement programmé de la population active [6]. Les nouvelles organisations du travail tendent à réduire les marges de manœuvre dont disposent les travailleurs pour faire face aux contraintes, et fragilisent les salariés souffrant de TMS. L'individualisation des relations de travail et la dislocation des collectifs de travail qui en découle, signifie en effet la perte des capacités collectives de régulation des contraintes: les individus se retrouvent souvent seuls pour faire face aux situations de travail, à leurs contraintes et aléas, et sont de fait fragilisés.

Le réseau pilote de surveillance épidémiologique des TMS mis en place par l'Institut de Veille Sanitaire en 2002 dans la région des Pays de la Loire apporte des informations inédites en France sur les TMS [12]. L'objectif de cet article est de synthétiser les résultats concernant la prévalence dans la population salariée des symptômes musculosquelettiques et des TMS diagnostiqués, ainsi que de leurs principaux facteurs de risque, et d'en discuter la portée dans une perspective de santé publique. L'analyse des modèles de risque de TMS et leurs perspectives ergonomiques à proprement parler ne seront pas abordés dans cet article.

\section{Méthode}

La surveillance épidémiologique des TMS repose sur un réseau sentinelle de 83 médecins du travail volontaires, représentant $18 \%$ des médecins du travail de la région des Pays de la Loire et présentant des caractéristiques d'exercice professionnel similaires à celles des médecins du travail de la région. Les salariés ont été inclus lors de la visite médicale périodique entre 2002 et 2004, selon une procédure de tirage au sort parmi l'effectif surveillé 
par chaque médecin sentinelle qui s'est effectuée en deux temps : sélection par le médecin de demi-journées d'étude, puis de un salarié parmi 10 vus au cours de cette période. Chaque année, les médecins devaient inclure 15 à 30 salariés selon qu'ils travaillaient à temps partiel ou à temps plein. Au total, 3710 salariés âgés de 20 à 59 ans (2 162 hommes et 1548 femmes, âge moyen $=38,4 \pm 10,3$ ans) ont été inclus entre 2002 et 2004. La plupart des secteurs d'activités couverts par la médecine du travail sont correctement représentés dans l'échantillon et la distribution des sujets en fonction de l'âge est comparable à celle des actifs de la région pour les deux sexes, les 50-59 ans représentant $17 \%$ des hommes $(n=377)$ et $16 \%$ des femmes $(n=270)$. La sélection aléatoire des salariés et la taille importante de l'effectif sélectionné ont permis d'obtenir un échantillon globalement représentatif des salariés des entreprises privées et publiques des Pays de la Loire [12]. On observe néanmoins une légère sous-représentation des femmes qui s'explique par une sous-représentation des secteurs de la santé et de l'éducation dans notre échantillon.

Les symptômes musculosquelettiques des membres et du rachis ont été recueillis à l'aide d'un auto-questionnaire dérivé du questionnaire "Nordique " [12]. Les six principaux TMS des membres supérieurs (TCR, épicondylite latérale, tendinites des fléchisseurs ou des extenseurs des doigts, ténosynovite de De Quervain, SCC, syndrome du tunnel cubital) ont été diagnostiqués cliniquement par les médecins du travail. L'examen clinique a appliqué la démarche diagnostique du consensus européen "SALTSA » pour la surveillance épidémiologique des TMS [14]. Ce protocole décrit de manière rigoureuse les manœuvres cliniques standardisées et les arbres décisionnels pour le diagnostic des six principaux TMS des membres supérieurs. Tous les médecins du réseau ont été préalablement formés à la théorie et à la pratique de la démarche diagnostique.

L'évaluation de l'exposition professionnelle et des antécédents médicaux a été effectuée à l'aide d'un auto-questionnaire portant sur les facteurs biomécaniques (répétitivité, force, postures extrêmes) et psychosociaux de risque de TMS. Les trois aspects psychosociaux explorés, à savoir la demande psychologique, la latitude décisionnelle et le soutien social au travail, ont été évalués à l'aide du " Job Content Questionnaire » de Karasek dans sa version validée en français [9].

La prévalence des pathologies dans l'échantillon, qui représente la proportion de cas de TMS au moment de l'étude, a été calculée par sujets, et non par pathologies. Des scores d'exposition au risque de TMS ont été calculés selon les recommandations du consensus européen par sommation du nombre de facteurs de risque [14]. L'exposition professionnelle a été classée faible ou en "zone verte ", modérée ou en "zone jaune », élevée ou en "zone rouge », selon que le salarié était exposé respectivement à aucun, un ou au moins deux facteurs de risque.

\section{Résultats}

Les résultats du réseau présentés ici concernent, dans un premier temps, les prévalences des TMS diagnostiqués notamment en fonction de l'âge. Ils sont complétés ensuite par les prévalences des facteurs d'exposition ce qui 
permet d'établir un parallèle entre niveau d'exposition et pathologies relevées à des âges différents.

\section{Prévalence des six principaux TMS des membres supérieurs diagnostiqués}

$\mathrm{Au}$ moins un des six principaux TMS a été diagnostiqué au cours de l'examen clinique chez $15 \%$ des femmes et $11 \%$ des hommes, tous âges confondus (tableaul). Les tendinopathies de la coiffe des rotateurs (TCR) sont les plus fréquentes, devant le SCC et l'épicondylite latérale. La prévalence des TMS augmente avec l'ancienneté au poste de travail, mais la différence n'est pas significative après ajustement sur l'âge. La prévalence des TMS augmente significativement avec l'âge pour les deux sexes (tableau I), même après ajustement sur l'ancienneté au poste de travail $(p<0,05)$. Chez les 50-59 ans, $19 \%$ des hommes et $27 \%$ des femmes souffrent d'au moins un des six TMS. L'augmentation avec l'âge est particulièrement nette pour les TCR puisque leur fréquence augmente, entre 20-29 ans et 50-59 ans, de $2 \%$ à $11 \%$ chez les hommes et de $3 \%$ à $15 \%$ chez les femmes.

La prévalence des TMS est inégalement répartie en fonction des secteurs d'activité et des catégories socioprofessionnelles. Les secteurs d'activité les plus touchés sont, d'une part, les secteurs industriels (notamment l'automobile, les industries des biens intermédiaires et des biens de consommation, l'agroalimentaire) et, d'autre part, l'agriculture, les transports et l'administration. Les catégories ouvrières, notamment les ouvriers non qualifiés de l'industrie, les ouvriers agricoles et les manutentionnaires, sont les plus touchées par les TMS pour les deux sexes,

Tableau I: Prévalence des TMS des membres supérieurs diagnostiqués lors de l'examen clinique standardisé

\begin{tabular}{|c|c|c|c|c|c|c|c|c|c|c|}
\hline \multirow[b]{2}{*}{ Prévalence (\%) } & \multicolumn{2}{|r|}{$20-29$ ans } & \multicolumn{2}{|r|}{ 30-39 ans } & \multicolumn{2}{|r|}{ 40-49 ans } & \multicolumn{2}{|r|}{ 50-59 ans } & \multicolumn{2}{|r|}{ Ensemble } \\
\hline & $N$ & $\%$ [IC $95 \%]$ & $N$ & $\%$ [IC $95 \%]$ & $N$ & $\%$ [IC $95 \%]$ & $N$ & $\%$ [IC $95 \%]$ & $N$ & $\%$ [IC $95 \%]$ \\
\hline \multicolumn{11}{|l|}{ Hommes } \\
\hline $\begin{array}{l}\text { Tendinites } \\
\text { de l'épaule }\end{array}$ & 8 & $1,6[0,5 ; 2,6]$ & 34 & $5,2[3,5 ; 6,9]$ & 58 & $9,5[7,2 ; 11,8]$ & 42 & $11,1[8,0 ; 14,3]$ & 142 & $6,6[5,5 ; 7,6]$ \\
\hline $\begin{array}{l}\text { Épicondylite } \\
\text { latérale }\end{array}$ & 3 & $0,6[0 ; 1,3]$ & 7 & $1,1[0,3 ; 1,9]$ & 20 & $3,5[2,0 ; 5,0]$ & 21 & $6,0[3,5 ; 8,5]$ & 51 & $2,5[1,8 ; 3,1]$ \\
\hline $\begin{array}{l}\text { Syndrome du } \\
\text { canal carpien }\end{array}$ & 4 & $0,8[0 ; 1,5]$ & 17 & $2,6[1,4 ; 3,8]$ & 15 & $2,5[1,2 ; 3,7]$ & 14 & $3,7[1,8 ; 5,6]$ & 50 & $2,3[1,7 ; 3,0]$ \\
\hline $\begin{array}{l}\text { Au moins un } \\
\text { des six TMS }\end{array}$ & 22 & $4,3[2,5 ; 6,0]$ & 57 & $8,8[6,6 ; 10,9]$ & 90 & $14,7[11,9 ; 17,5]$ & 73 & $19,4[15,4 ; 23,4]$ & 242 & $11,2[9,9 ; 12,6]$ \\
\hline \multicolumn{11}{|l|}{ Femmes } \\
\hline $\begin{array}{l}\text { Tendinites } \\
\text { de l'épaule }\end{array}$ & 9 & $2,5[0,9 ; 4,1]$ & 23 & $5,4[3,2 ; 7,5]$ & 59 & $12,1[9,2 ; 15,0]$ & 41 & $15,2[10,9 ; 19,5]$ & 132 & $8,5[7,1 ; 9,9]$ \\
\hline $\begin{array}{l}\text { Épicondylite } \\
\text { latérale }\end{array}$ & 2 & $0,6[0 ; 1,3]$ & 9 & $2,2[0,8 ; 3,6]$ & 16 & $3,6[1,9 ; 5,3]$ & 12 & $5,0[2,2 ; 7,7]$ & 39 & $2,7[1,9 ; 3,5]$ \\
\hline $\begin{array}{l}\text { Syndrome du } \\
\text { canal carpien }\end{array}$ & 3 & $0,8[0 ; 1,8]$ & 15 & $3,5[1,8 ; 5,2]$ & 23 & $4,7[2,8 ; 6,6]$ & 21 & $7,8[4,6 ; 11,0]$ & 62 & $4,0[3,0 ; 5,0]$ \\
\hline $\begin{array}{l}\text { Au moins un } \\
\text { des six TMS }\end{array}$ & 17 & $4,7[2,5 ; 6,9]$ & 48 & $11,2[8,2 ; 14,2]$ & 92 & $18,9[15,4 ; 22,4]$ & 72 & $26,7[21,4 ; 31,9]$ & 229 & $14,8[13,0 ; 16,6]$ \\
\hline
\end{tabular}


quel que soit l'âge, devant les employés. Chez les 50-59 ans, $30 \%$ des ouvriers et $34 \%$ des ouvrières présentent un TMS cliniquement diagnostiqué, principalement une TCR (22\% des ouvriers et $17 \%$ des ouvrières).

\section{Prévalence de l'exposition au risque de TMS}

La surveillance épidémiologique des TMS dans les Pays de la Loire fournit une première description de l'exposition aux facteurs de risque de TMS des membres supérieurs à l'échelle d'une région. La majorité des salariés des deux sexes est fortement exposée à ces facteurs de risque, qu'ils soient de nature biomécanique, psychosociale et organisationnelle. L'intensité de l'exposition aux facteurs de risque de TMS du membre supérieur (nuque exceptée) varie avec l'âge. Les salariés les plus jeunes (classe d'âges de 20-29 ans) sont les plus exposés : $71 \%$ d'entre eux sont exposés à deux facteurs de risque ou plus, contre $66 \%$ des 30-39 ans, $64 \%$ des 40-49 ans et $61 \%$ des 50-59 ans. La surexposition au risque de TMS chez les 20-29 ans concerne majoritairement les hommes (76\%) et s'explique principalement par la surexposition des personnels intérimaires.

L'étude montre cependant que l'exposition professionnelle reste élevée après 50 ans, quel que soit le sexe. Ceci a été observé pour la plupart des facteurs de risque biomécaniques et psychosociaux considérés individuellement (tableau II). Ainsi, entre 50 et 59 ans, $21 \%$ des hommes et $31 \%$ des femmes étaient exposés à des gestes répétitifs plus de 4 heures par

Tableau II: Prévalence de l'exposition aux principaux facteurs de risque biomécaniques et psychosociaux de TMS de la nuque et des membres supérieurs en fonction de l'âge

\begin{tabular}{|l|c|c|c|c|c|c|c|c|}
\hline \multirow{2}{*}{ Exposition élevée (\%) } & \multicolumn{5}{|c|}{ Hommes } & \multicolumn{4}{c|}{ Femmes } \\
\cline { 2 - 10 } & \multicolumn{3}{|c|}{$<50$ ans } & \multicolumn{2}{|c|}{$\geq 50$ ans } & \multicolumn{2}{|c|}{$<50$ ans } & \multicolumn{2}{c|}{$\geq 50$ ans } \\
\hline \multicolumn{1}{|c|}{ Facteurs de risque } & $N$ & $\%[I C 95 \%]$ & $N$ & $\%[I C 95 \%]$ & $N$ & $\%[I C 95 \%]$ & $N$ & $\%[I C 95 \%]$ \\
\hline Répétitivité élevée & 398 & $22,5[20,5 ; 24,4]$ & 78 & $20,9[16,8 ; 25,0]$ & 399 & $31,5[29,0 ; 34,1]$ & 82 & $31,2[25,6 ; 36,8]$ \\
\hline Force élevée & 814 & $50,2[47,7 ; 52,6]$ & 161 & $47,6[42,3 ; 53,0]$ & 324 & $29,1[26,4 ; 31,8]$ & 59 & $26,2[20,5 ; 32,0]$ \\
\hline $\begin{array}{l}\text { Bras en abduction } \\
>90^{\circ}\end{array}$ & 242 & $13,6[12,0 ; 15,2]$ & 62 & $16,7[12,9 ; 20,5]$ & 155 & $12,2[10,4 ; 14,0]$ & 26 & $9,7[6,2 ; 13,3]$ \\
\hline Torsion du poignet & 645 & $36,6[34,4 ; 38,9]$ & 101 & $27,4[22,9 ; 32,0]$ & 411 & $32,5[29,9 ; 35,1]$ & 75 & $28,4[23,0 ; 33,8]$ \\
\hline $\begin{array}{l}\text { Charge physique } \\
\text { lourde (Borg >15) }\end{array}$ & 413 & $23,2[21,3 ; 25,2]$ & 90 & $23,9[19,6 ; 28,2]$ & 243 & $19,0[16,9 ; 21,2]$ & 56 & $20,7[15,9 ; 25,6]$ \\
\hline $\begin{array}{l}\text { Demande } \\
\text { psychologique élevée }\end{array}$ & 507 & $28,5[26,4 ; 30,6]$ & 100 & $26,5[22,1 ; 31,0]$ & 372 & $29,1[26,6 ; 31,6]$ & 68 & $25,2[20,0 ; 30,4]$ \\
\hline $\begin{array}{l}\text { Latitude décisionnelle } \\
\text { faible }\end{array}$ & 973 & $54,7[52,4 ; 57,0]$ & 209 & $55,4[50,4 ; 60,5]$ & 820 & $64,2[61,6 ; 66,8]$ & 187 & $69,3[63,8 ; 74,8]$ \\
\hline Soutien social faible & 461 & $25,9[23,9 ; 27,9]$ & 96 & $25,5[21,1 ; 29,9]$ & 328 & $25,7[23,3 ; 28,1]$ & 81 & $30,0[24,5 ; 35,5]$ \\
\hline Tension au travail & 228 & $12,8[11,3 ; 14,4]$ & 49 & $13,0[9,6 ; 16,4]$ & 224 & $17,5[15,5 ; 19,6]$ & 38 & $14,1[9,9 ; 18,2]$ \\
\hline $\begin{array}{l}\text { Polyvalence } \\
\text { quotidienne }\end{array}$ & 413 & $23,3[21,4 ; 25,3]$ & 106 & $28,4[23,8 ; 33,0]$ & 306 & $24,2[21,8 ; 26,5]$ & 46 & $17,4[12,8 ; 21,9]$ \\
\hline Horaires irréguliers & 638 & $36,0[33,8 ; 38,3]$ & 127 & $33,9[29,1 ; 38,7]$ & 430 & $33,9[31,3 ; 36,5]$ & 80 & $30,1[24,6 ; 35,6]$ \\
\hline
\end{tabular}

* Définition des facteurs de risque in $[8,9]$. 
jour, $48 \%$ des hommes et $26 \%$ des femmes manipulaient des objets lourds, $17 \%$ des hommes et $10 \%$ des femmes travaillaient avec les bras au-dessus de l'horizontale, $27 \%$ des hommes et $28 \%$ des femmes travaillaient avec le poignet en position inconfortable. Concernant l'exposition des plus de 50 ans aux facteurs psychosociaux, $55 \%$ des hommes et $69 \%$ des femmes manquaient de latitude décisionnelle, $25 \%$ des hommes et $30 \%$ des femmes manquaient de soutien social de la part de leur encadrement ou de leurs collègues, $13 \%$ des hommes et $14 \%$ des femmes étaient en situation de tension au travail (conjugaison d'une demande psychologique forte et d'une latitude décisionnelle faible). La charge de travail globale évaluée à l'aide de l'échelle psychophysique de Borg était élevée (score $\geq 15$ ) pour $24 \%$ des hommes et $21 \%$ des femmes de 50-59 ans. Le niveau de l'exposition aux facteurs de risque de TMS était particulièrement élevé (exposition à deux facteurs de risque ou plus) pour les catégories ouvrières et, à un moindre degré, pour les employés. Il était moins élevé pour les professions intermédiaires et pour les cadres. Après 50 ans, $75 \%$ des ouvriers et $71 \%$ des ouvrières restaient exposés à au moins deux des facteurs de risque de TMS.

\section{Discussion}

La région des Pays de la Loire retenue pour la surveillance épidémiologique des TMS est proche pour de nombreux paramètres de la France métropolitaine. La principale singularité des Pays de la Loire est l'importance du secteur industriel et, par conséquent, du nombre d'ouvriers industriels. Ceci doit être pris en considération dans l'extrapolation des résultats du réseau expérimental à d'autres régions françaises du fait du risque élevé de TMS dans ces secteurs et catégories d'emplois, pouvant majorer l'estimation de la prévalence des TMS par rapport à celles observées dans des régions moins industrielles [12].

La surveillance épidémiologique des TMS est basée sur un large réseau régional de 83 médecins sentinelles dont les caractéristiques professionnelles étaient comparables à ceux qui n'ont pas participé. L'inclusion aléatoire des salariés lors de la visite médicale assure une représentativité satisfaisante de l'échantillon par rapport à la population salariée régionale [12], ce qui permet une meilleure estimation de la prévalence des TMS dans la population salariée que les estimations réalisées par des récentes études françaises conduites dans des secteurs à haut risque de TMS [7]. Ce type d'approche pourrait être complété par des études plus fines, notamment ergonomiques, afin de prendre en compte la singularité des situations de travail en entreprises [2].

Les résultats montrent l'importance de la prévalence des TMS diagnostiqués par le médecin du travail chez les salariés en activité, puisque près de $13 \%$ d'entre eux présentent au moins un TMS des membres supérieurs. Contrairement aux statistiques d'indemnisation des maladies professionnelles, le TMS le plus fréquemment observé n'est pas le SCC, mais la TCR et ce, quels que soient la classe d'âge et le sexe. 
La prévalence des TMS diagnostiqués cliniquement augmente nettement avec l'âge, même après ajustement sur l'ancienneté au poste de travail. Ceci confirme des études menées en France et dans les pays anglo-saxons en population salariée ou en population générale $[7,8,10]$. Néanmoins, il est difficile de faire la part des choses entre l'avancée en âge et le cumul des expositions professionnelles, l'ancienneté au poste différant de l'ancienneté d'exposition et ne renseignant par sur les parcours professionnels des salariés [8]. Quoi qu'il en soit, l'étude montre la très forte prévalence des TMS après 50 ans. En effet, bien que ces salariés âgés soient tous employés au moment de l'enquête, un quart présente cliniquement au moins un des six TMS, notamment une TCR ou un SCC, et $6 \%$ présente deux TMS ou plus. Cette accumulation de TMS chez les salariés vieillissants diminue leurs capacités fonctionnelles et augmente le risque d'incapacité de travail et de licenciement [11].

Les secteurs d'activité les plus touchés sont, d'une part, les secteurs industriels et l'agriculture et, d'autre part, l'administration. Ceci est en accord, d'une manière globale, avec les grandes enquêtes américaines et européennes $[8,10]$. Les ouvriers non qualifiés du secteur industriel sont les plus touchés, devant les manutentionnaires, les personnels de services directs aux particuliers et les ouvriers agricoles.

L'étude montre que la majorité de salariés des deux sexes est fortement exposée aux facteurs de risque biomécaniques, psychosociaux et organisationnels de TMS. D'une manière générale, le niveau de l'exposition aux facteurs de risque de TMS est particulièrement élevé pour les catégories ouvrières et, à un moindre degré, pour les employés. Les résultats montrent que le niveau d'exposition professionnelle au risque de TMS ne diminue pas après 50 ans alors que les capacités fonctionnelles diminuent à cet âge. Ceci est vrai pour l'ensemble des principaux facteurs de risque tels que la répétitivité des gestes, la force, les postures inconfortables. Le manque de latitude décisionnelle pour faire face aux contraintes de la tâche concerne une majorité de salariés de 50 ans et plus : plus d'un homme sur deux et sept femmes sur dix. Il peut réduire la capacité des salariés souffrant des TMS à répondre aux contraintes des situations qu'ils maîtrisaient auparavant, et conduire à un risque d'exclusion des plus âgés [2,5]. Ce constat inquiétant montre le manque de prise en considération de la pénibilité du travail des salariés vieillissants et les carences de la prévention primaire des TMS.

\section{Développer une politique de santé au travail}

Les TMS participent aux fortes inégalités sociales de santé au travail en France en raison de la surexposition des ouvriers et des employés. Toutes les conditions sont actuellement réunies pour que les difficultés de maintien en emploi des salariés les plus fragilisés et les plus exposés à la concurrence mondiale, débordent le dispositif actuel de santé au travail et rendent caduques les objectifs d'accroissement du taux d'activité des seniors en France. Ces processus d'exclusion ne sont donc pas seulement des drames humains et sociaux; ils sont source de pertes de compétitivité des entreprises françaises en raison des coûts qu’ils génèrent [1]. La réponse aux difficultés de maintien en emploi d'un travailleur confronté à un handicap de situation généré par la diminution de ses capacités fonctionnelles pour faire face aux contraintes de son poste de travail (par exemple, ne pas pouvoir 
porter une charge lourde ou élever le bras au-delà de l'horizontale), ne peut donc pas être seulement individuelle et rétroactive mais doit être collective et proactive. Le processus d'exclusion des salariés présentant un handicap de situation nécessite une réflexion approfondie et un changement d'échelle dans la politique de maintien en emploi des salariés et ceci indépendamment de l'issue de la réflexion réglementaire sur l'aptitude/inaptitude médicale au travail. Les stratégies de maintien en emploi ne doivent pas être vues comme un simple dispositif palliatif à la décision d'inaptitude mais intégrées dans la réflexion sur les parcours professionnels. En effet, elles ne relèvent pas seulement d'une approche biomédicale du handicap de situation, mais nécessitent une réflexion approfondie sur l'organisation du travail et le travail soutenable tout au long de la vie, y compris les aspects relatifs à la mobilité professionnelle et à la formation au cours de la vie dont sont souvent exclus les ouvriers et les employés peu qualifiés $[2,4,6]$. Des expériences étrangères [8], notamment québécoises, comme l'assignation temporaire et le retour thérapeutique au travail par exemple, montrent que des stratégies en réseau sont pertinentes à condition de lever les obstacles réglementaires entre médecine de soins, de réadaptation, de prévention et d'assurance. À ce titre, la complexité de la réglementation et de la structuration du processus de maintien en emploi reste un obstacle important à l'heure actuelle en France, malgré la nouvelle loi sur le handicap. La mise en place de la pluridisciplinarité dans les services médicaux du travail est un premier élément de réponse encore insuffisant. Reste à développer l'élaboration collective des plans d'activité et des règles de métiers intégrant les différents partenaires de la pluridisciplinarité. Une coordination des intervenants et la mise en place de réseaux et de systèmes proactifs impliquant les entreprises et permettant d'accompagner précocement les travailleurs en situation de handicap relatif semblent être des pistes de réflexion intéressantes.

Prévenir les TMS et maintenir en emploi les salariés, nécessite d'amplifier les actions de prévention des TMS dans les entreprises pour réduire l'exposition au risque et prendre en compte les situations de handicap des salariés souffrant de TMS $[4,6,12,13]$. Cela nécessite une forte mobilisation de l'ensemble des acteurs de la prévention des risques professionnels (entreprises, partenaires sociaux, pouvoirs publics) et la mise en place d'une politique structurée et coordonnée de prévention des TMS dans les entreprises et dans les services de santé au travail[6]. Bien qu'incomplètes, les connaissances scientifiques actuelles sont suffisantes pour agir, comme le montrent des expériences d'accompagnement ergonomiques en entreprises [5].

\section{Conclusion}

La surveillance des TMS dans les entreprises des Pays de la Loire montre que les salariés vieillissants, comme les salariés plus jeunes, sont exposés à des degrés d'intensité divers à un cumul de contraintes biomécaniques, psychosociales et organisationnelles. La forte prévalence des TMS après 50 ans conjuguée à des contraintes importantes, souligne la nécessité d'une approche globale de la prévention des TMS dans les entreprises et une réflexion sur des conditions de travail soutenable tout au long de la vie. 


\section{REMERCIEMENTS}

Nous remercions les médecins du travail et les services de santé au travail des Pays de la Loire qui ont participé à cette étude : Docteurs Abonnat, Banon, Bardet, Benetti, Becquemie, Bertin, Bertrand, Bidron, Biton, Bizouarne, Boisse, Bonamy, Bonneau, Bouguer, Bouguer-Diquelou, Bourut-Lacouture, Breton, Caillon, Cesbron, Chisacof, Chotard, Compain, Coquin-Georgeac, Cordes, Couet, Coutand, Danielou, Darcy, Davenas, De Lescure, De Lansalut, Dopsent, Dupas, Evano, Fache, Fontaine, Frampas-Chotard, Guillier, Guillimin, Harinte, Harrigan, Hervio, Hirigoyen, Jahan, Joliveau, Jube, Kalfon, Laine-Colin, Laventure, Le Dizet, Lechevalier, Le Clerc, Ledenvic, Leroux, Leroy-Maguer, Levrard, Levy, Logeay, Lucas, Mallet, Martin, Mazoyer, Meritet, Michel, Migne-Cousseau, Moisan, Page, Patillot, Pinaud, Pineau, Pizzala, Plessis, Plouhinec, Raffray, Robin, Roussel, Russu, Saboureault, Schlindwein, Soulard, Thomson, Treillard, Tripodi.

\section{BIBLIOGRAPHIE}

1. Askenazy P. Les désordres du travail. Le seuil, « la République des idées », Paris, 2004.

2. Bourgeois F, Lemarchand C, Hubault F et al. Troubles musculosquelettiques et travail. Quand la santé interroge l'organisation. Editions de l'ANACT, Lyon, 2006.

3. Caisse nationale de l'assurance maladie des travailleurs salariés. Statistiques nationales des accidents du travail, des accidents de trajet et des maladies professionnelles (année 2003), Paris, 2005.

4. Caroly, S, Coutarel, F, Escriva, E, Roquelaure, Y, Schweitzer, JM, Daniellou F. La prévention durable des TMS : quels freins, quels leviers d'action ? Rapport de recherche 120 p. + annexes, Paris : Direction Générale du Travail, Ministère de l'emploi, de la cohésion sociale et du logement. (A paraître 2008).

5. Escriva E. Agir sur les situations de handicap : le maintien dans l'emploi. Editions de l'ANACT, 2004.

6. Lasfargues G, Roquelaure Y, Fouquet B, Leclerc A. Pathologies ostéoarticulaires par hypersollicitation d'origine professionnelle, Masson, Paris, 2003, 141 p.

7. Leclerc A, Landre MF, Chastang JF, Niedhammer I, Roquelaure Y. A longitudinal study on upper limb disorders in repetitive work. Scand J Work Environ Health 2001;27:268-78.

8. National research council. The National Academy of Sciences. Musculoskeletal Disorders and the Workplace: Low back and Upper Extremity musculoskeletal disorders. National Academy Press, Washington, DC, 2001.

9. Niedhammer I. Psychometric properties of the French version of the Karasek Job Content Questionnaire : a study of the scales of decision latitude, psychological demands, social support, and physical demands in the GAZEL cohort. Int Arch Occup Environ Health 2002;75:129-44.

10. Paoli P, Merllié $D$. Troisième enquête sur les conditions de travail: dix ans de conditions de travail dans l'Union européenne. European foundation for the improvement of living and working conditions, Dublin, 2000. http://www.eurofound.eu.int/publications/files/EF0121EN.

11. Roquelaure Y, Cren S, Rousseau F, Touranchet A, Dano C, Fanello S, Penneau-Fontbonne D. Work status after workers' compensation claims for upper limb musculoskeletal disorders. Occup Environ Med 2004; 61:79-81.

12. Roquelaure $Y$, Ha C, Leclerc $A$, Touranchet $A$, Sauteron $M$, Imbernon $M$, Goldberg $M$ and 80 occupational physicians of the Pays de la Loire region. Epidemiological Surveillance of Upper Extremity Musculoskeletal Disorders in the Working Population: the French Pays de la Loire Study. Arthritis Rheum 2006; 55:765-78.

13. Roquelaure Y. Workplace intervention and musculoskeletal disorders: the need to develop research on implementation strategy. Occup Environ Med 2008;65: 4-5.

14. Sluiter JK, Rest KM, Frings-Dresen MHW. Criteria document for evaluating the work-relatedness of upper extremity musculoskeletal disorders. Scand J Work Environ Health 2001;27 suppl 1:1-102. 